\title{
Processing nanoparticle-nanocarbon composites as binder-free electrodes for lithium-based batteries
}

\author{
Marya Baloch $^{2,3} \cdot$ Pierre Kubiak $^{1}$ - Vladimir Roddatis ${ }^{4} \cdot$ Oleksandr Bondarchuk $^{2}$ • \\ Carmen M. López ${ }^{1}$ (1)
}

Received: 16 May 2017/ Accepted: 20 September 2017 / Published online: 27 September 2017

(c) The Author(s) 2017. This article is an open access publication

\begin{abstract}
The processing of battery materials into functional electrodes traditionally requires the preparation of slurries using binders, organic solvents, and additives, all of which present economic and environmental challenges. These are amplified in the production of nanostructured carbon electrodes which are often more difficult to disperse in slurries and require more energy-intensive and longer processing. In this study we demonstrate a new process for preparing binder-free nanocarbon/nanoparticle (Fe-C) composite electrodes and study the effect of processing on the nanocomposite's cycling performance in lithium cells. The binder-free electrodes were prepared by a two-step
\end{abstract}

method: pulsed-electrodeposition of iron-based catalyst followed by chemical vapor deposition of a carbon film. SEM and TEM of the Fe-C showed that the active materials have a fibrous and tortuous morphology with disordered nanocrystalline domains characteristic of an amorphous carbon. The $\mathrm{Fe}-\mathrm{C}$ electrodes showed good mechanical stability and an excellent cycle performance with an average stable capacity of $221 \mathrm{mAhg}^{-1}$, and $85 \%$ capacity retention for up to 50 cycles. By reducing the number of processing steps and eliminating the use of binders and other chemicals this new method offers a "greener" alternative than current processing methods.

Electronic supplementary material The online version of this article (doi:10.1007/s40243-017-0105-5) contains supplementary material, which is available to authorized users.

Carmen M. López

cLopez@hbku.edu.qa; padmanirv@gmail.com

1 Qatar Environment and Energy Research Institute (QEERI), HBKU, Qatar Foundation, P.O. Box 34110, Doha, Qatar

2 CICenergigune, Albert Einstein 48, 01510 Miñano, Alava, Spain

3 Departamento de Química Inorgánica, Universidad del País Vasco UPV/EHU, Apdo. 644, 48080 Bilbao, Spain

4 Institut für Materialphysik, Universität Göttingen, FriedrichHund-Platz 1, 37077 Göttingen, Germany 


\section{Graphical abstract}

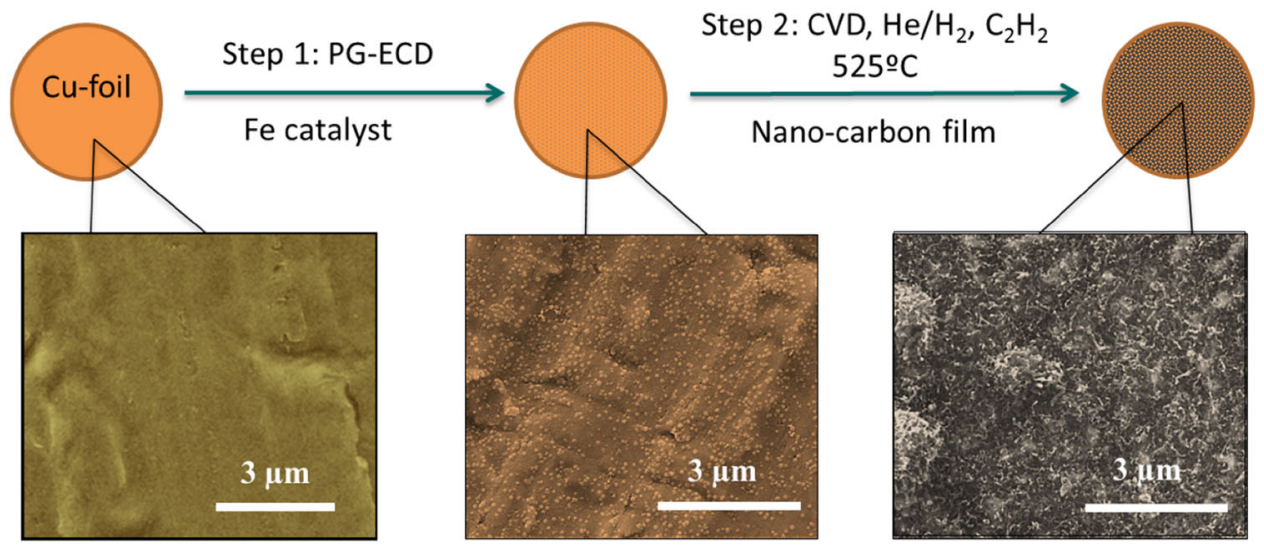

Synopsis: gains in sustainability can be achieved by eliminating use of binders, chemicals, and the number of electrode's processing steps in this new method.

Keywords Binder-free electrodes · Nanocarbons . Electrode processing $\cdot$ CVD $\cdot$ Composites

\section{Introduction}

Advanced batteries such as Li-ion and other Li-based batteries are widely recognized as crucial enabling technologies in the race to implement sustainable and renewable sources of energy. Within battery development, electrode formulation and processing are often overlooked yet critical steps in the production of materials for advanced batteries. Well-established methods for commercially available materials are usually based on slurry preparation with the active, a polymeric binder, a conductive additive, and a solvent that can serve as good dispersion medium for all the components [1-3]. Scalable and compatible with large-scale manufacturing practices, this "traditional" method produces electrodes with good thermal and chemical stability as well as excellent mechanical and electrical properties. However, this type of processing tends to be long, with various energy-consuming steps and the use of organic solvents, all of which poses significant economic and environmental challenges [1, 2]. In addition to organic media, aqueous-based binders can also be used to formulate electrodes; the extreme sensitivity of most lithium-based battery systems to trace amounts of water has slowed down their large-scale adoption, however [1-3].

Despite great promise, nanocarbonaceous electrodes have not yet met with the expected commercial success due, in part, to increased complexity and cost during electrode processing. Filamentous nanocarbons-whether graphitic or amorphous-are usually prepared by high energy methods such as chemical vapor deposition (CVD) pyrolysis, arc-discharge, etc., after which they are removed from the growth substrate and treated with acidic solutions before a slurry can be prepared $[1,4-6]$. Nanoparticulate matter, however, is much more difficult to disperse in slurry than other commercial battery materials; this is specially challenging for filamentous nanocarbons $[1,3,7,8]$. Another challenge for these materials is that their increased surface areas and surface defects make them more susceptible to side reactions and uncontrolled growth of the solid electrolyte interphase layer (SEI). In Li-ion batteries, this growth is the main contributor to lithium-ion inventory loss and raise in interphasial resistance of anodes which limits the life cycle of the cells $[1,4,8,9]$. And strategies to avoid these problems are not usually readily available or easy to implement.

In this work we demonstrate the production of binderfree nanocarbonaceous electrodes by a "greener" method that reduces the number of processing steps and the use and volume of chemicals. When dispensing with binders, having a good intrinsic contact between the growth substrate and the carbon is critical as the substrate itself becomes the current collector. Binder-free nanocarbonaceous electrodes can result in substantial sustainability gains and yet, very few examples have been reportedpossibly due to the weak adhesion of nanocarbons to their growth substrates $[8,10-13]$. To achieve this critical contact we have chosen to use electrodeposition to prepare the Fe-base catalysts for nanocarbon synthesis. Electrodeposition as a method of preparing thin nanoparticulate films has many advantages which include, among others, high purity of the electrodeposits, high yield, and good control over the size, orientation and distribution of particles across the conductive substrate [14-16]. In particular, pulsed 
techniques allow for the easy preparation of smaller nanoparticle clusters suitable for filamentous growth $[14,17,18]$. After PG-ECD the electrodes are transferred to a CVD reactor for the final processing step of nanocarbon synthesis.

The electrodes we obtained had sufficient mechanical stability to be used directly in a lithium half-cell without any further treatment. They showed good electrochemical properties and cycling stability that compared favorably with previously reported disordered carbons [6, 19-22]. Additionally, we found that the role of the incorporated $\mathrm{Fe}$ based nanoparticles in the electrochemical performance was due to $\mathrm{Fe}_{3} \mathrm{C}$ species which were passivated during the first few charge-discharge cycles.

\section{Experimental}

Self-standing nanocarbon-nanoparticle electrodes (Fe-C) were prepared by a two-step method. Step 1: pulsed-galvanostatic electrodeposition (PG-ECD) of iron-based growth catalyst and, step 2: chemical vapor deposition (CVD) of nanocarbon fibers. Unless otherwise indicated, all materials and solvents were used as received, without further purification, and stored in an argon atmosphere glove box $\left(\mathrm{O}_{2}, \mathrm{H}_{2} \mathrm{O} \leq 2 \mathrm{ppm}\right)$. SEM images were collected in a FEI Quanta 200 FEG scanning electron microscope, operating at $18 \mathrm{keV}$. TEM images were collected in a FEI Tecnai G2 instrument operating at $200 \mathrm{keV}$.

\section{PG-ECD of Fe-growth catalyst}

PG-ECD of the iron growth catalyst has been performed according to the method published before with some modifications [14]. All electrochemical experiments have been carried out in an extraction hood. Formamide (spectrophotometric grade $\geq 99 \%$, Sigma-Aldrich) was used to prepare the plating bath (i.e., electrodeposition solution) and was opened and stored exclusively in an Ar-filled glove box. $\mathrm{FeCl}_{2}$ was used right after purchase and stored in a desiccator. All the solutions used for PG-ECD were prepared fresh immediately before each experiment, and were not reused afterwards. Plating solution: $0.01 \mathrm{M}$ $\mathrm{FeCl}_{2} / 0.1 \mathrm{M} \mathrm{LiClO}_{4} /$ formamide $\left(\mathrm{LiClO}_{4}\right.$ as supporting electrolyte). The electrolysis cell was a three-electrode beaker cell $(10 \mathrm{~mL})$, and deposition was done using a VMP 5-channel Potentiostat/Galvanostat (Bio Logic). The working electrode (battery grade copper foil, Goodfellow, pre-cleaned with ethanol) was a circular piece $\left(A=1.539 \mathrm{~cm}^{2}\right)$ fitted with a copper tape connection and insulated on one face with kapton tape. The reference electrode was an $\mathrm{Ag} / \mathrm{AgCl}$, in $3 \mathrm{M} \mathrm{KCl}$. The counter electrode was $\mathrm{Pt}$ on glass prepared as described in the supporting information file. PG-ECD parameters: current density $i=-1.62 \mathrm{mAcm}^{-2}$, time on $\left(T_{\mathrm{ON}}\right): 0.001 \mathrm{~s}$, time off $\left(T_{\mathrm{OFF}}\right): 0.5 \mathrm{~s}, 500$ pulses. After $\mathrm{Fe}$ deposition the samples were rinsed with de-ionized water (Millipore Milli-Q system), and acetone to remove the Kapton tape, glue residues, and the contact. Then they were rinsed again with de-ionized water and dried with an air-gun.

\section{CVD of nanocarbons}

CVD synthesis of the self-standing films has been performed according to the method described before [12]. The nanocarbon electrodes were prepared from $20 \mathrm{sccm}$ (standard cubic centimeters per minute) of acetylene $\left(\mathrm{C}_{2} \mathrm{H}_{2}\right)$ gas diluted in a mixture of $70 \mathrm{v} \%$ of helium $(\mathrm{He}, 700 \mathrm{sccm})$ as carrier gas, and $30 \mathrm{v} \%$ of hydrogen $\left(\mathrm{H}_{2}, 300 \mathrm{sccm}\right)$ at $525-575{ }^{\circ} \mathrm{C}$, for $10 \mathrm{~min}$. After cooling the materials where characterized by SEM and TEM.

\section{Electrochemical evaluation}

Preliminary electrochemical evaluation of the binder-free nanocarbon-nanoparticle films has been performed in lithium half-cells. Coin cells (Hohsen CR 2016 cell hardware) were prepared using lithium foil (Goodfellow), the $\mathrm{Fe}-\mathrm{C}$ composite electrode, Celgard 2325 separators, and 1.2 $\mathrm{M} \mathrm{LiPF}_{6} /$ ethylene carbonate (EC):dimethyl carbonate (DMC) (30:70 wt\%, UBE Corp.) as the electrolyte. The mass loading of $\mathrm{Fe}-\mathrm{C}$ composite was $0.0714 \mathrm{mgcm}^{-2}$ for a $\mathrm{Cu}$-foil circular electrode $14 \mathrm{~mm}$ in diameter. The mass loading of Fe-based nanoparticles was $0.06461 \mathrm{mgcm}^{-2}$ for the same electrode's size. The cells were cycled galvanostatically between 0 and $2 \mathrm{~V}$ vs. $\mathrm{Li}^{\prime} / \mathrm{Li}^{+}$with a cycling current of $2.2 \mu \mathrm{Ag}^{-1}$ (normalized to the mass of $\mathrm{Fe}-\mathrm{C}$ or Fe-nanoparticles as appropriate).

\section{Results and discussion}

\section{Processing and morphology}

Preparation of the binder-free $\mathrm{Fe}-\mathrm{C}$ electrodes was achieved by the two-step processing method illustrated schematically on Fig. 1. Step 1 is the preparation of an iron-based nanoparticle film by PG-ECD directly into the surface a copper current collector (Fig. 1a). Using PG-ECD serves a dual purpose; first, and most important, it allows for the $\mathrm{Fe}$ nanoparticles to a have a good contact with the conductive substrate (Fig. 2a-c). This is crucial to get binder-free electrodes with sufficient adhesion to the substrate to insure that the $\mathrm{Fe}-\mathrm{C}$ film obtained in step 2 has good electrical contact to the current collector for battery performance. Second, using PG-ECD, small and well-distributed

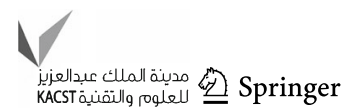




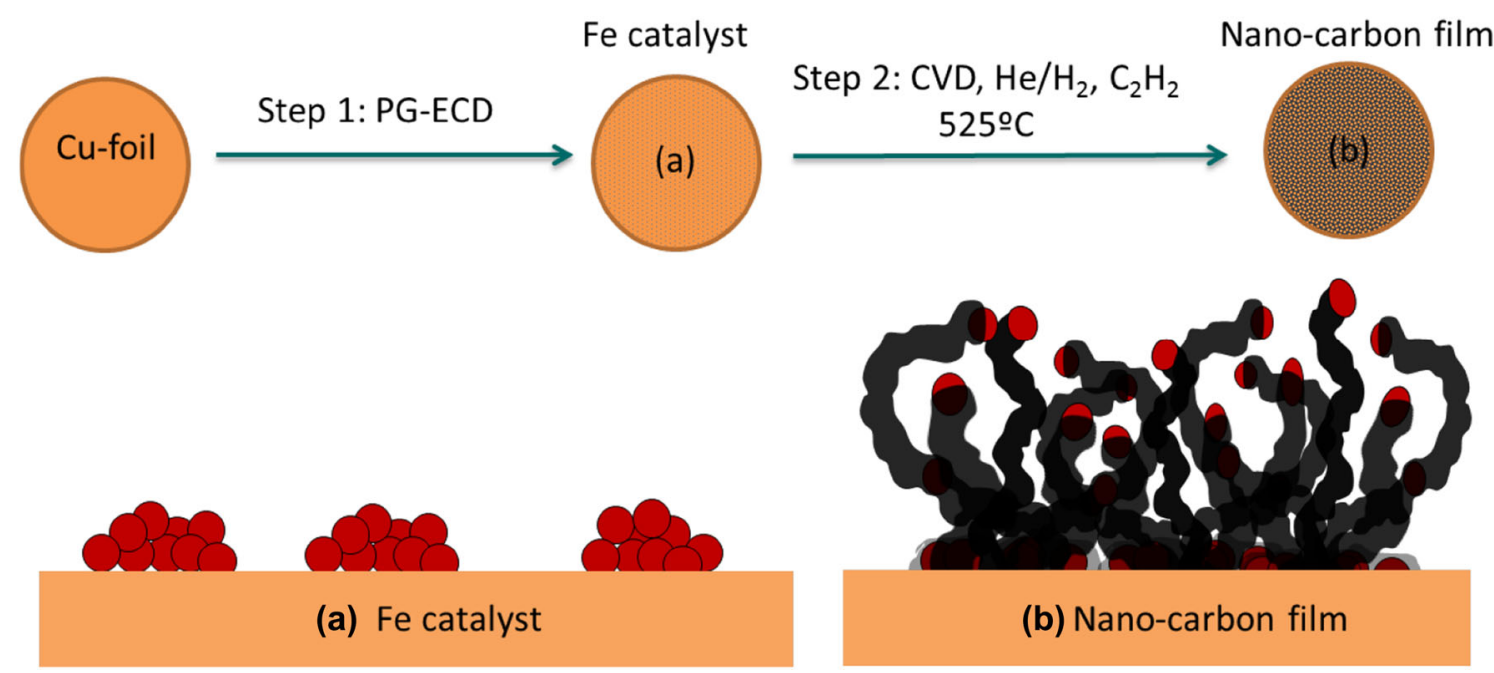

Fig. 1 Preparation of nano-carbon self-standing films by the two-step method of PG-ECD followed by CVD: a Fe-nanoparticles electrodeposited onto $\mathrm{Cu}$-foil, and $\mathbf{b} \mathrm{Fe}-\mathrm{C}$ composite electrode

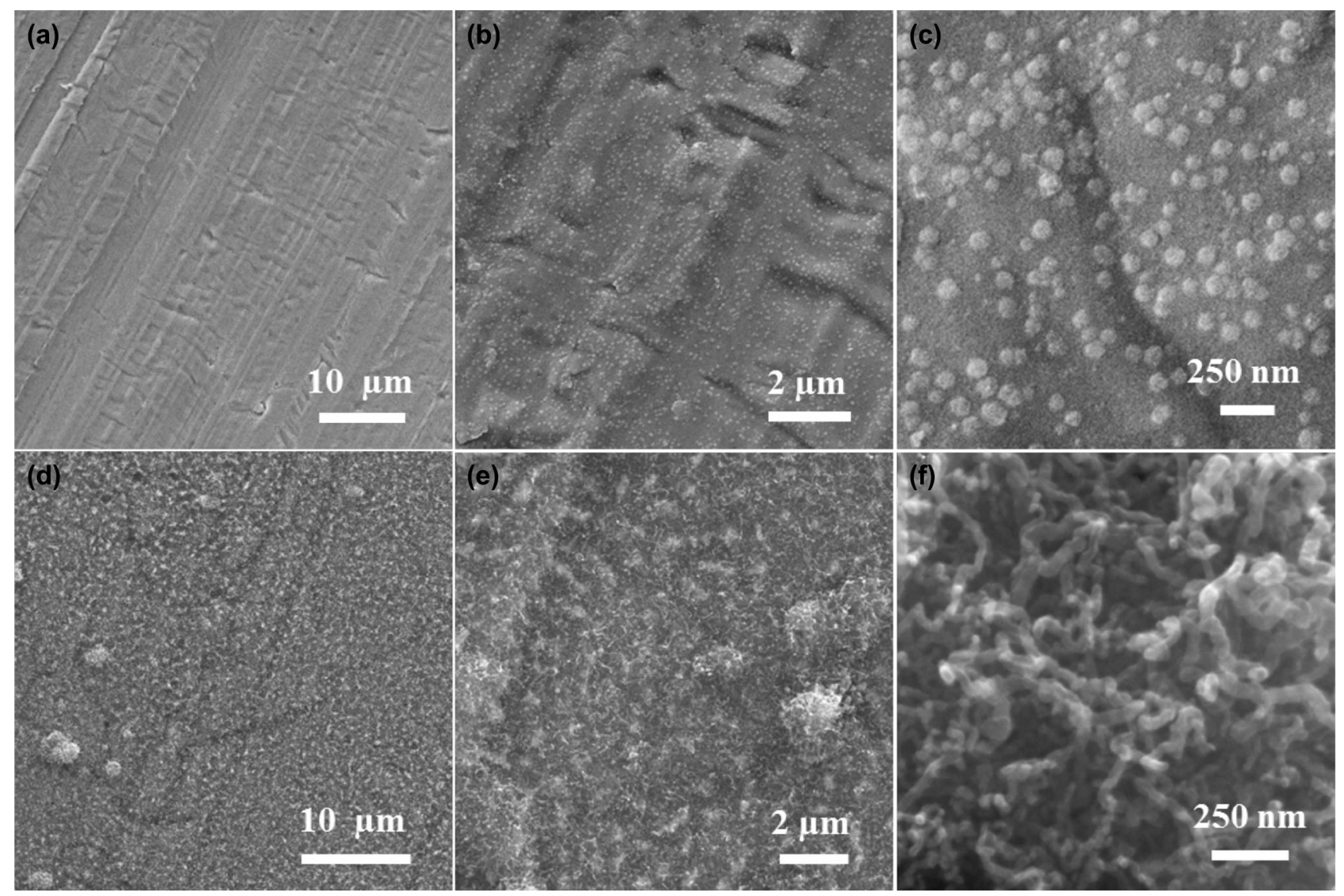

Fig. 2 Morphology of the Fe-growth catalyst and nanocarbon-nanoparticle composites. SEM of as-deposited Fe-catalyst film at different magnifications (a-c), and nanocarbon-nanoparticle composite electrodes at different magnifications $(\mathbf{d}-\mathbf{f})$

nanoparticle clusters can be obtained (Figs. 1a, 2a-c), as it is well known that the diameter of fibrous nanocarbons is affected by the size of the catalytic particles. In step 2, this electrode is used as substrate to grow nanocarbon fibers through CVD. Figure S2 shows photographs of two nanocarbonaceous electrodes produced by our method and of commercial amorphous carbon produced by the traditional slurry method. The nanocarbonaceous electrodes 

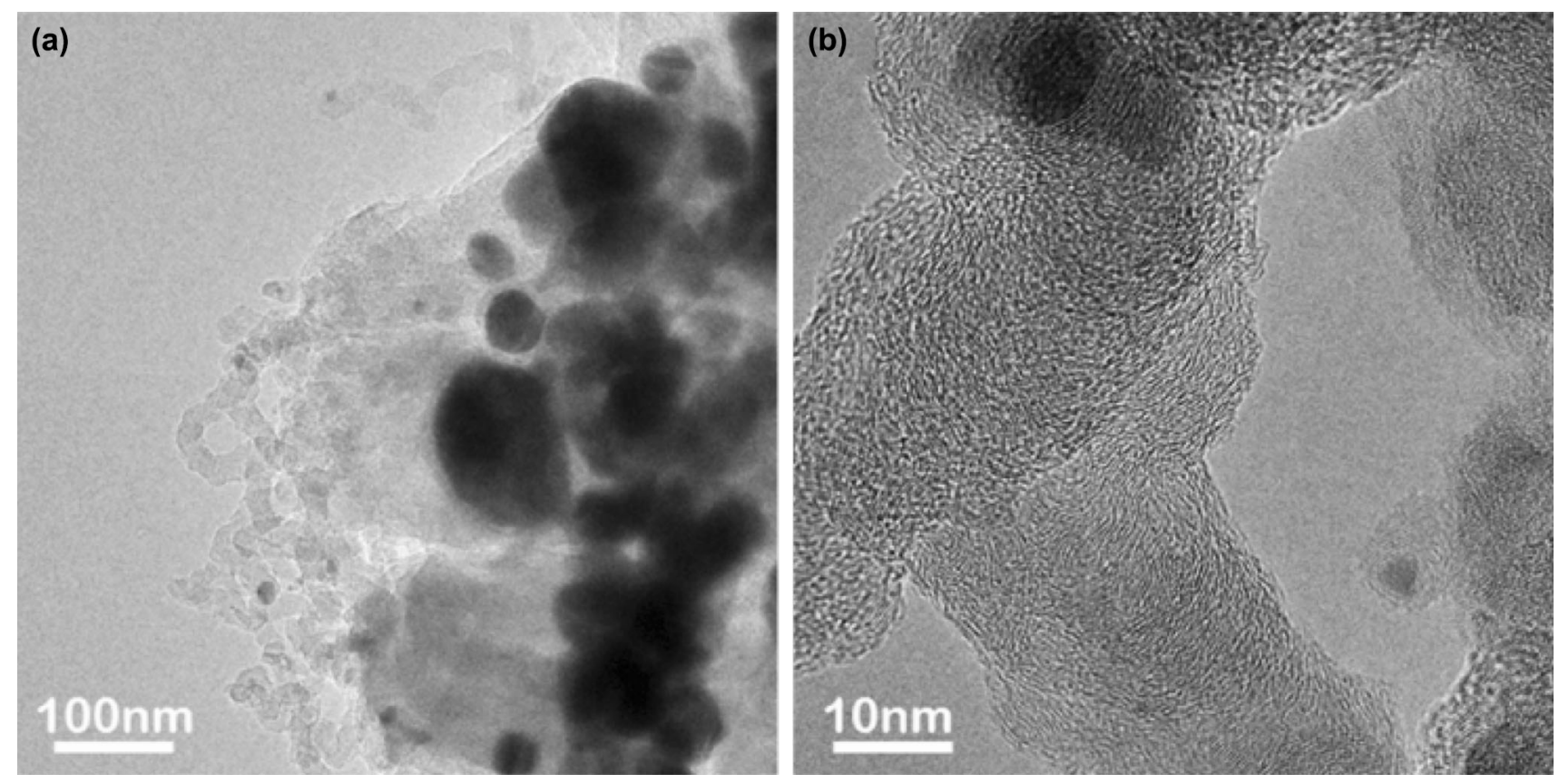

Fig. 3 Structural characterization by TEM: a lower magnification showing aggregates and fibrous features, b HRTEM showing the disordered nanocrystalline structure

show uniform coverage of the current collector comparable with the traditionally prepared one. A side by side comparison of our method and "traditional" slurry preparation (table S1, supporting info.) shows the typical number of steps in each type of process; this can give a fair idea of how much simpler our method is.

In principle, any metal or oxide nanoparticles that can catalyze the growth of nanocarbons could be used with our method, provided than it can be deposited with good enough contact to produce a binder-free film that doesn't easily fall off the electrode after CVD. For this proof of concept study we chose Fe because it is the most common growth catalyst used; metallic Fe itself is not electrochemically active towards lithium but the electrochemical behavior of its oxides and hydroxides is very well known and documented. Other possible growth catalysts could include traditional ones such as $\mathrm{Ni}, \mathrm{Co}, \mathrm{Cu}$, manganese oxides, and some more recently reported such as $\mathrm{MgAl}$ oxides and oxyhydroxides, or $\mathrm{TiO}_{2}[4,5,10,12]$.

The morphology of the Fe-carbon nano-composite electrodes is shown in Fig. 1e-h. The films are typically composed of multiple fibers evenly distributed in an open fibrous network, and coverage that matches closely the topology of the substrate (Fig. 1e-h). The average thickness is approximately $1 \mu \mathrm{m}$, while a few scattered taller clusters of fibers can also be observed (Fig. 1e, f). The individual fibers have an average diameter in the range of $20-60 \mathrm{~nm}$, with a tortuous morphology that results from tip growth (Figs. 2h, 3). This tortuous fiber morphology suggests a certain level of turbulent flow of the gaseous medium at the substrate/gas interphase. The transmission electron microscopy (TEM) images presented in Fig. 3 also show the morphology of the $\mathrm{Fe}-\mathrm{C}$ films after CVD. Two types of Fe-clusters can be observed: (1) bigger aggregates (100-500 $\mathrm{nm}$ dia.) that do not produce nanocarbon fibers and (2) smaller aggregates (20-60 nm dia.) which produce nanocarbon fibers, (Fig. 3a). The growth process during CVD poses a trade-off between substrate-catalyst interaction and filament growth. Electrodeposited Fe-particles can grow by aggregation during CVD. Those sitting at the bottom of the electrodeposit become sheathed in disordered nanocarbon but cannot be pushed by the growth to make fibers [type (1)]; these clusters provide adhesion to the current collector. Particles that sit higher in the electrodeposit undergo less self-aggregation and less interaction with the current collector and can be pushed up to direct the growth of the $\mathrm{Fe}-\mathrm{C}$ fibers [type (2)]. In Fig. 3b, the high resolution TEM shows that the structure of the fibers is comprised of disordered nano-crystalline domains with an interlayer distance of $0.36-0.38 \mathrm{~nm}$, which is compatible with disordered carbons and larger than the interlayer distance of graphite $(0.34 \mathrm{~nm})$. Both, structural disorder and larger interlayer distance have been identified as factors that can improve $\mathrm{Li}^{+}$and $\mathrm{Na}^{+}$ion storage in carbonaceous electrodes [6, 21, 22].

\section{Electrochemical performance}

The efficacy of our method in producing disordered nanocarbon electrodes was tested by evaluating their electrochemical performance in lithium cells. These

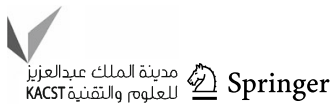



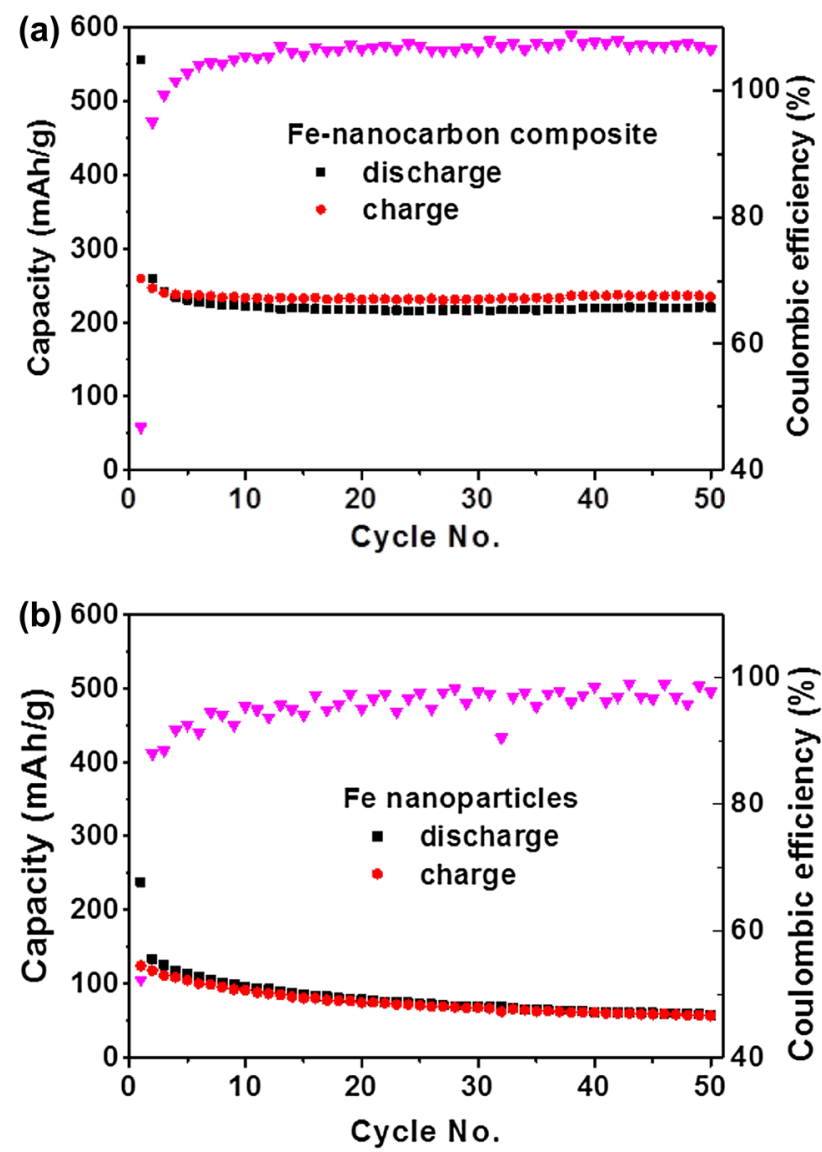

Fig. 4 Cycling performance on a lithium half-cell of: a $\mathrm{Fe}-\mathrm{C}$ composite electrode, and $\mathbf{b}$ as-deposited Fe-based nanoparticle electrode

electrodes contain no binders, no additional conductive additives and had not being otherwise treated or purified to remove metal growth catalysts, therefore, their performance mostly depends on the quality of the films themselves. Figures 4 and 5 show the cycling performance, galvanostatic curves, and differential capacity curves of the $\mathrm{Fe}-\mathrm{C}$ produced by our method. We have also carried-out the electrochemical evaluation of as-deposited Fe-based nanoparticle electrodes to identify their contribution to the electrochemical performance of the composite films.

A trade-off between making the process greener and the effect of having Fe-nanoparticles incorporated into the electrode has to be carefully considered. It is well known that the high surface area of the Fe-nanoparticles causes them to react immediately with the atmosphere to form a thin native oxide layer. Iron species such as $\mathrm{Fe}_{2} \mathrm{O}_{3}, \mathrm{Fe}_{3} \mathrm{O}_{4}$, and $\mathrm{FeOOH}$ can react electrochemically with $\mathrm{Li}^{+}$providing extra capacity but suffering from pulverization and side reactions with the electrolyte [23-25]. $\mathrm{Fe}-\mathrm{C}$ nano-composites (Fig. 4a) show good capacity and a remarkable cycling stability for a binder-free nanocarbonaceous electrode. The composite exhibits a large first discharge capacity of
$556 \mathrm{mAh} / \mathrm{g}$ and a first reversible capacity of $260 \mathrm{mAh} / \mathrm{g}$ which leads to an irreversible capacity of $296 \mathrm{mAh} / \mathrm{g}$. The first cycle irreversible capacity of the $\mathrm{Fe}-\mathrm{C}$ electrodes is characteristic of filamentous nanostructured carbons, whether graphitic or disordered, and it is due to the influence of the high surface area on the formation and aging of the solid electrolyte interphase (SEI) layer [4-8, 28]. After the first cycle it maintains a reversible capacity of more than $220 \mathrm{mAh} / \mathrm{g}$ for 50 cycles without capacity fading, $85 \%$ capacity retention. The galvanostatic curves shown in Fig. 5a do not show any plateau due to iron oxides or oxyhydroxides which suggests that all $\mathrm{Fe}^{2+/ 3+}$ species of the native oxide layer have been reduced during the CVD process. In fact, the major iron-containing species in the composite exhibits electrochemical performance compatible with $\mathrm{Fe}_{3} \mathrm{C}$, as demonstrated by the first discharge peaks at 1.01 and $0.62 \mathrm{~V}$ in the $\mathrm{d} Q / \mathrm{d} V$ curves (Fig. $5 \mathrm{~b}$ ). These peaks are in good agreement to those reported previously by other workers $[8,26,27]$. As cycling progresses the peak at $0.62 \mathrm{~V}$ disappears completely and the peak at $1.01 \mathrm{~V}$ becomes significantly smaller and shifts to close to $0.95 \mathrm{~V}$. This shift is attributed to passivation of some surface sites by formation of the SEI layer in the nanocomposite electrode. Furthermore, the shape of the $\mathrm{d} Q / \mathrm{d} V$ suggests that the capacity observed is due to lithium-ion storage in amorphous carbon, although the precise mechanism is not yet clear. The origin of the higher than a $100 \%$ coulombic efficiency for the $\mathrm{Fe}-\mathrm{C}$ electrodes probably originates from the various non-Faradaic processes associated to SEI formation, conversion reactions of the native oxide layer of the Fe-nanoparticles, and capacitive and pseudocapacitive effects due to the disordered nature of the carbon nano-domains. The precise nature of these processes will be investigated in future studies.

The cycling performance of the as-deposited Fe-nanoparticles is shown in Fig. 4b. A first discharge capacity of $238 \mathrm{mAh} / \mathrm{g}$ and a first reversible capacity of $125 \mathrm{mAh} / \mathrm{g}$ can be observed, which leads to an irreversible capacity of 113 $\mathrm{mAh} / \mathrm{g}$. The capacity decreases rapidly upon cycling and only $75 \mathrm{mAh} / \mathrm{g}$ can be achieved after 20 cycles and $58 \mathrm{mAh} / \mathrm{g}$ after 50 cycles, representing only $46 \%$ capacity retention. Moreover, a rather low coulombic efficiency $(<98 \%)$ is also observed for this electrode. The poor stability of $\mathrm{Fe}$ nanoparticles can also be seen on the galvanostatic chargedischarge curves in Fig. 5c. The first discharge is characterized by three pseudo-plateaus at $0.12,0.80$ and $1.23 \mathrm{~V}$ vs. Li/ $\mathrm{Li}^{+}$. The higher and lower voltage plateaus appear to be irreversible whereas the intermediate one (at $0.80 \mathrm{~V}$ ) progressively disappears upon cycling; it still can be observed on 3rd and 10th cycle but not on 20th cycle. These indicate that the 0.12 and $0.80 \mathrm{~V}$ plateaus most probably correspond to electrolyte decomposition and other SEI-forming processes, and that the higher voltage plateau is probably due to the 

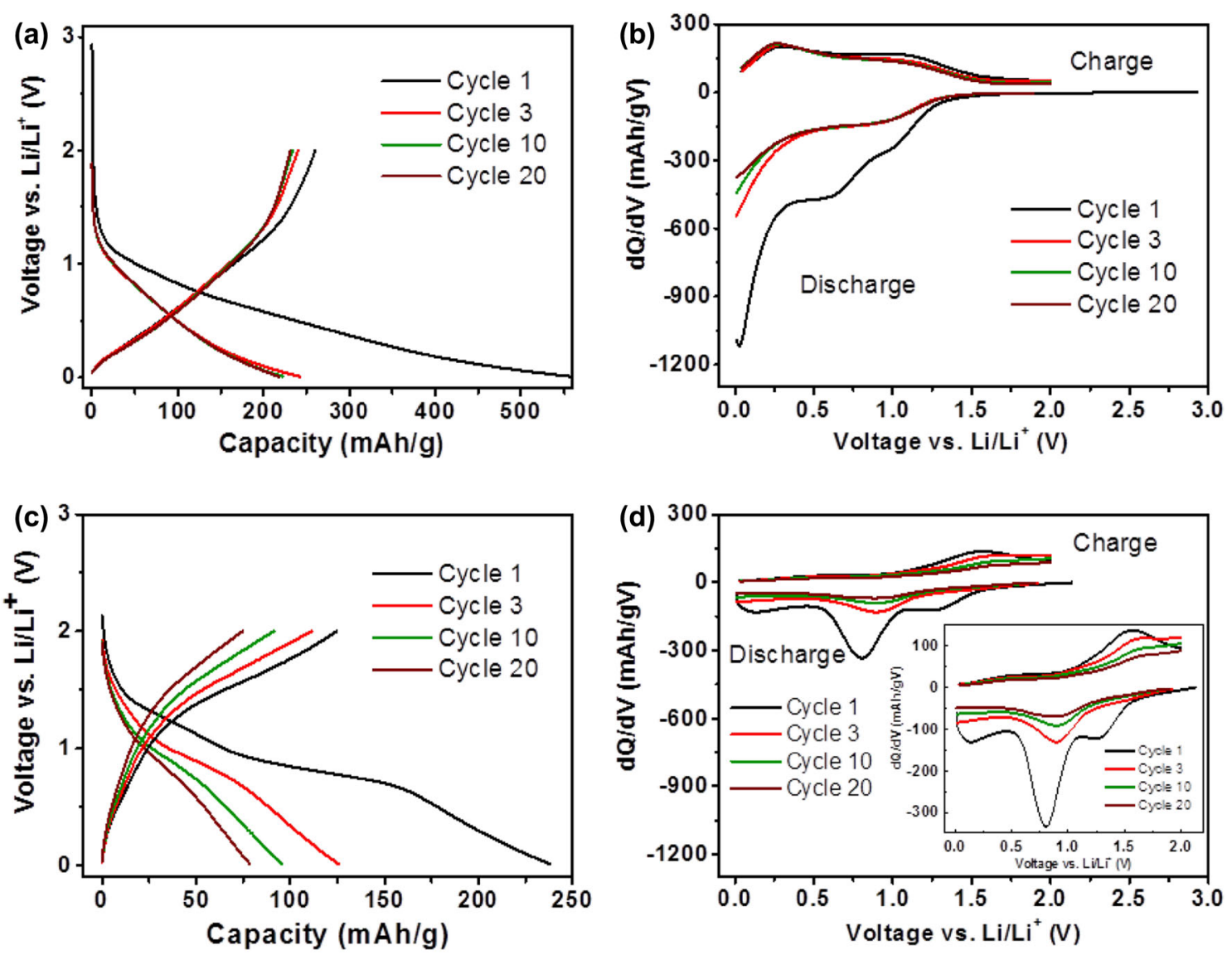

Fig. 5 Electrochemical behavior of the nanostructured electrodes. Galvanostatic curves of: a Fe-nanocarbon composite electrode, and c as-deposited Fe nanoparticle electrode. Differential capacity curves

electrochemical activity of iron-containing species that lose activity with cycling. The differential capacity $(\mathrm{d} Q / \mathrm{d} V)$ curves in Fig. 5d confirm the previous observations. The peaks at 1.23 and $0.80 \mathrm{~V} \mathrm{vs}$. $\mathrm{Li} / \mathrm{Li}^{+}$of the first cycle in the $\mathrm{d} Q / \mathrm{d} V$ curve are characteristic of the reduction of iron oxides $\left(\mathrm{Fe}_{2} \mathrm{O}_{3}\right.$, $\mathrm{Fe}_{3} \mathrm{O}_{4}$ ) and oxyhydroxides $(\mathrm{FeOOH})$ in good agreement with which has been previously reported [23-25]. The electrochemical behavior of the as-deposited Fe-nanoparticles films is significantly different from that of the $\mathrm{Fe}-\mathrm{C}$ films, which confirms our previous observations regarding the performance of the composite.

\section{Conclusion}

In this work we demonstrated a new preparation method (consisting of PG-ECD, CVD) for mechanically stable, binder-free $\mathrm{Fe}$-nanocarbon composites. This method produced electrodes composed of $\mathrm{Fe}_{3} \mathrm{C}$ nanoparticles embedded in a matrix of disordered nanocrystalline carbon with predominantly fibrous morphology. We measured a favorable electrochemical performance compatible with reversible

of: b Fe-nanocarbon composite electrode, and $\mathbf{d}$ as-deposited $\mathrm{Fe}$ nanoparticle electrode. The insert in $\mathbf{d}$ is a blow-up of the same differential capacity curve

$\mathrm{Li}^{+}$storage mainly in the nano-carbon domains. Additionally, we found that the formation of the SEI interphase in these films is mainly affected by the iron-containing species $\mathrm{Fe}_{3} \mathrm{C}$, which indicates a pathway for future work in tailoring the relative population of these species to enhance performance. By reducing the use of extra chemicals and the number of processing steps this new method presents a significant advantage in sustainability when compared with traditional slurry-based electrode processing.

Acknowledgements MB thanks Dr. Javier Carretero-González for training in the use of the CVD reactor. Funding by the Department of Industry, Innovation, Commerce and Tourism of the Basque Government through the ETORTEK program is gratefully acknowledged.

Open Access This article is distributed under the terms of the Creative Commons Attribution 4.0 International License (http:// creativecommons.org/licenses/by/4.0/), which permits unrestricted use, distribution, and reproduction in any medium, provided you give appropriate credit to the original author(s) and the source, provide a link to the Creative Commons license, and indicate if changes were made. 


\section{References}

1. Kraytsberg, A., Ein-Eli, Y.: Conveying advanced Li-ion battery materials into practice. The impact of electrode slurry preparation skills. Adv. Energy Mater. (2016). doi:10.1002/aenm.201600655

2. Wu, Q., Ha, S., Prakash, J., Dees, D.W., Lu, W.: Investigation on high energy lithium-ion batteries with aqueous binders. Electrochim. Acta 114, 1-6 (2013)

3. Chou, S.L., Pan, L., Wang, J.Z., Liu, H.K., Dou, S.X.: Small things make a big difference: binder effects on the performance of $\mathrm{Li}$ and $\mathrm{Na}$ batteries. Phys. Chem. Chem. Phys. 16, 20347-20359 (2014)

4. Liu, X.M., Huang, Z.D., Oh, S.W., Zhang, B., Ma, P.C., Yuen, M.M.F., Kim, J.K.: Carbon nanotube (CNT)-based composites as electrode material for rechargeable $\mathrm{Li}$-ion batteries: a review. Compos. Sci. Technol. 72, 121-144 (2012)

5. Kumar, M.: Carbon nanotubes synthesis and growth mechanisms. In: Yellampalli, S. (ed.) Carbon nanotubes-synthesis, characterization, applications. InTech Europe, Rijeka (2011)

6. Tang, J., Etacheri, V., Pol, V.G.: Wild fungus derived carbon fibers and hybrid as anodes for lithium-ion batteries. ACS Sustain. Chem. Eng. 4(5), 2624-2636 (2016)

7. Varzi, A., Täubert, C., Wohlfahrt-Mehrens, M.: The effects of pristine and carboxylated multi-walled carbon nanotubes as conductive additives on the performance of $\mathrm{LiNi}_{0.33} \mathrm{Co}_{0.33}$ $\mathrm{Mn}_{0.33} \mathrm{O}_{2}$ and $\mathrm{LiFePO}_{4}$ positives electrodes. Electrochim. Acta 78, 17-26 (2012)

8. Zhao, X., Xia, D., Yue, J., Liu, S.: In-situ generated nano-Fe ${ }_{3} \mathrm{C}$ embedded into nitrogen-doped carbon for high performance anode in lithium ion battery. Electrochim. Acta 116, 292-299 (2014)

9. Sarasketa-Zabala, E., Aguesse, F., Villareal, I., Rodriguez-Martinez, L.M., López, C.M., Kubiak, P.: Understanding lithium inventory loss and sudden performance fade in cylindrical cells during cycling with deep-discharge steps. J. Phys. Chem. C 119, 896-906 (2015)

10. Cheng, Y., Chen, Z., Zhu, M., Lu, Y.: Polyacrylic acid assisted assembly of oxide particles and carbon nanotubes for high-performance flexible battery anodes. Adv. Energy Mater. (2015). doi:10.1002/aenm.201401207

11. Nair, J.R., Rius, G., Jagadale, P., Destro, M., Tortello, M., Yoshimura, M., Tagliaferro, A., Gerbaldi, C.: Remarkably stable high power Li-ion battery anodes based on vertically arranged multilayered-graphene. Electrochim. Acta 182, 500-506 (2015)

12. Carretero-González, J., Pérez-Villar, S., Roddatis, V.V., Gómez, N., Bondarchuk, O.B., Lopatin, S., López, C.M.: In-situ generation of metal-metal oxide catalysts for the growth of highly oriented graphitic nanowiggles. Carbon 68, 821-825 (2014)

13. Huggins, T.M., Whiteley, J.M., Love, C.T., Lee, K., Lee, S.H., Ren, Z.J., Biffinger, J.C.: Controlled growth of nanostructured biotemplates with cobalt and nitrogen codoping as a binderless lithium-ion battery anode. ACS Appl. Mater. Interfaces 8(40), 26868-26877 (2016)
14. Baloch, M., López, C.M.: Effect of additives on the pulsed-galvanostatic electrodeposition of iron nanoparticles from formamide media. ChemElectroChem. 3, 883-886 (2016)

15. López, C.M., Choi, K.-S.: Electrochemical synthesis of dendritic zinc films composed of systematically varying motif crystals. Langmuir 22(25), 10625-10629 (2006)

16. Kang, D., Kim, T.W., Kubota, S.R., Cardiel, A.C., Cha, H.G., Choi, K.-S.: Electrochemical synthesis of photoelectrodes and catalysts for use in solar water splitting. Chem. Rev. 115, 12839-12887 (2015)

17. Pushpavanam, M., Chandrasekar, M.S.: Pulse and pulse reverse plating - conceptual advantages and applications. Electrochim. Acta 53, 3313-3322 (2008)

18. Tantavichet, N., Pritzker, M.: Low-and high-frequency pulse current and pulse reverse plating of copper. J. Electrochem. Soc. 150(10), C665-C677 (2003)

19. Ogihara, N., Igarashi, Y., Kamakura, A., Naoi, K., Kusachi, Y., Utsugi, K.: Disordered carbon negative electrode for electrochemical capacitors and high-rate batteries. Electrochim. Acta 52, 1713-1720 (2006)

20. Naoi, K., Ogihara, N., Igarashi, Y., Kamakura, A., Kusachi, Y., Utsugi, K.: Disordered carbon anode for lithium-ion battery. I. An interfacial reversible redox action and anomalous topology changes. J. Electrochem. Soc. 152(6), A1047-A1053 (2005)

21. Gong, J., Wu, H.: Electrochemical intercalation of lithium species into disordered carbon prepared by the heat-treatment of $\operatorname{poly}(p$ phenylene) at $650{ }^{\circ} \mathrm{C}$ for anode in lithium-ion battery. Electrochim. Acta 45, 1753-1762 (2000)

22. Tsai, P.-C., Chung, S.-C., Lin, S.-K., Yamada, A.: Ab initio study of sodium intercalation into disordered carbon. J. Mater. Chem. A 3, 9763-9768 (2015)

23. Lee, S.H., Yu, S.-H., Lee, J.E., Jin, A., Lee, D.J., Lee, N., Jo, H., Shin, K., Ahn, T.-Y., Kim, Y.-W., Choe, H., Sung, Y.-E., Hyeon, T.: Self-assembled $\mathrm{Fe}_{3} \mathrm{O}_{4}$ nanoparticle clusters as high-performance anodes for lithium ion batteries via geometric confinement. Nano Lett. 13, 4249-4256 (2013)

24. Wang, J., Zhou, H., Nanda, J., Braun, P.V.: Three-dimensionally mesostructured $\mathrm{Fe}_{2} \mathrm{O}_{3}$ electrodes with good rate performance and reduced voltage hysteresis. Chem. Mater. 27, 2803-2811 (2015)

25. Tabuchi, T., Katayama, Y., Nukuda, T., Ogumi, Z.: Surface reaction of $\beta-\mathrm{FeOOH}$ film negative electrode for lithium-ion cells. J. Power Sour 191, 636-639 (2009)

26. Su, L., Zhou, Z., Shen, P.: Core-shell $\mathrm{Fe} @ \mathrm{Fe}_{3} \mathrm{C} / \mathrm{C}$ nanocomposites as anode materials for $\mathrm{Li}$ ion batteries. Electrochim. Acta 87, 180-185 (2013)

27. Li, J., Wen, W., Xu, G., Zhou, M., Huang, Z., Guan, L.: Fe-added $\mathrm{Fe}_{3} \mathrm{C}$ carbon nanofibers as anode for $\mathrm{Li}$ ion batteries with excellent low-temperature performance. Electrochim. Acta 153, 300-305 (2015)

28. Xu, Z.-L., Yao, S., Cui, J., Zhou, L., Kim, J.-K.: Atomic scale, amorphous $\mathrm{FeOx} /$ carbon nanofiber anodes for $\mathrm{Li}$-ion and $\mathrm{Na}$-ion batteries. Eng. Storage Mater. 8, 10-19 (2017) 